\title{
Additive properties of random sequences of positive integers
}

by

P. ERdös and A. RÉNxI (Budapest)

§ 0. Introduction. It is well known (see e.g. [1]) that the number of those integers $n \leqslant x$ which can be represented in the form $n=k^{2}+l^{2}$ ( $k$ and $l$ integers) has the order of magnitude $x / \sqrt{\log x}$; as clearly the number of pairs $k, l$ of positive integers such that $k^{2}+l^{2} \leqslant x$ is $\sim \pi x / 4$, the reason why the set of numbers which can be represented as the sum of two squares has zero density is not that the squares are too rare, but loosely speaking - that they are "too regularly" distributed, so that among the sums $k^{2}+l^{2}$ there are too many equal ones. This was first demonstrated by Atkin [2], who solved the following problem, proposed by J. E. Littlewood: If each square $k^{2}$ is replaced by a random integer $v_{k}$, chosen according to a certain probability law in the neighbourhood of $k^{2}$, then the sums $v_{k}+\nu_{l}$ almost surely have a positive density.

In $\S 1$ of the present paper we introduce a class of sequences of random integers. This construction has been used already in [3]. In $\S 2,3$ and 4 we prove some theorems of a similar character to that of Atkin, mentioned above. We shall show that if the random sequences $v_{k}$ and $\mu_{l}$ have approximately the same order of magnitude as the sequence $c k^{2}$ with some $c>0$, then the sequences $v_{k}+\mu_{l}, k^{2}+\mu_{l}$ and $i_{k}+v_{l}$ will have positive density with probability 1 ; moreover, in all three cases the sequences of numbers $n$ which have exactly $r$ representations in the form $n=v_{k}+\mu_{l}, n=k^{2}+\mu_{l}$ or $n=v_{k}+v_{l}(k<l)$, will almost surely have a positive density for each value of $r(r=0,1, \ldots)$ and these densities form a Poisson distribution. In $\S 5$ we shall show that the number $f(n)$ of representations of $n$ in the form $n=v_{k}+v_{l}$ has, in case it tends to $+\infty$, a normal distribution in the limit. In $\S 6$ we generalize these results for sums of more than two terms of a random sequence of integers. In $\S 7$ we consider the distribution of differences of a random sequence. $\S 8$ deals with random sequences $\nu_{k}$ of the order $k^{2+\varepsilon}$, where $\varepsilon>0$ is arbitrarily small, for which the number $f(n)$ of representations of $n$ in the form 
$n=v_{k}+v_{l}$ is almost surely bounded. This result is connected with a problem of S. Sidon ([4], [5]). $\S 9$ deals with great values of $f(n)$ while $\S 10$ contains the proof of the strong law of large numbers for $f(n)$. Finally in $\$ 11$ we give a stochastic analogon of Romanoff's theorem [6], according to which the sequence of those numbers $n$ which can be represented in the form $n=p+a^{k}$, where $p$ is prime and $a>1$ an integer, has positive density, while $\S 12$ contains a similar but more general theorem.

Some of the results proved in detail in the present paper have been announced without proof in a previous paper [3] of the first-named author.

Throughout the paper we use the following notation: $P(\ldots)$ denotes the probability of the event in the brackets. We denote random events by capital letters; $\bar{A}$ denotes the event contrary to $A$; if $A$ and $B$ are events, let $A+B$ denote the event consisting in the occurrence of at least one of the events $A$ and $B$, and let $A B$ denote the event consisting in the joint occurrence of the events $A$ and $B$. We denote random variables by Greek letters $\xi, \eta, v, \mu$ etc. $M(\xi)$ denotes the mean value and $D^{2}(\xi)$ the variance of the random variable $\xi . M(\xi \mid A)$ denotes the conditional mean value of $\xi$ under condition $A . C_{1}, C_{2}, \ldots$ denote positive constants.

\$1. Random sequences of integers. The notations introduced in this section will be used throughout the paper.

We define the random sequences of positive integers dealt with in the present paper as follows: Let $\xi_{n}(n=1,2, \ldots)$ be a sequence of completely independent random variables such that $\xi_{n}$ takes on the values 1 and 0 with the corresponding probabilities $p_{n}$ and $\left(1-p_{n}\right)$, i. e. we suppose that

$$
P\left(\xi_{n}=1\right)=p_{n}, \quad P\left(\xi_{n}=0\right)=1-p_{n},
$$

where $p_{n}$ is an arbitrary sequence of numbers such that $0 \leqslant p_{n} \leqslant 1$ and

$$
\sum_{n=1}^{\infty} p_{n}=+\infty \text {. }
$$

We denote by $v_{1}, v_{2}, \ldots, v_{k}, \ldots$ the values of $n$ (in increasing order of magnitude) such that $\xi_{n}=1$. Thus $\nu_{1}<v_{2}<\ldots<v_{k}<\ldots, \xi_{v_{k}}=1$. $(k=1,2, \ldots)$ and $\xi_{n}=0$ if $v_{k}<n<v_{k+1}$. We call the sequence $\left\{v_{k}\right\}$ a random sequence of positive integers generated by the sequence $\left\{p_{n}\right\}$ of probabilities. $p_{n}$ is clearly the probability that the number $n$ should be contained in the sequence $\left\{v_{k}\right\}$. This method of generating random sequences of integers has already been used in [3]. Evidently ordinary sequences of integers are special cases of random sequences of the above type, which we obtain if each $p_{n}$ is 1 or 0 . Clearly we have

$$
\xi_{1}+\xi_{2}+\ldots+\xi_{v_{k}}=k \quad(k=1,2, \ldots)
$$

and $v_{k}$ is the least integer for which (1.3) holds for a given value of $k$. It follows by the Lemma of Borel-Cantelli (see [9]) from (1.2) that the sequence $\left\{v_{k}\right\}$ is infinite with probability 1 . It follows further from a variant of the strong law of large numbers (see [10], p. 438) that putting

$$
P(n)=\sum_{k=1}^{n} p_{k}
$$

we have with probability 1

$$
\lim _{n \rightarrow+\infty} \frac{\xi_{1}+\xi_{2}+\ldots+\xi_{n}}{P(n)}=1 .
$$

Thus by (1.3) and (1.5) we have with probability 1

$$
\lim _{k \rightarrow+\infty} \frac{k}{P\left(v_{k}\right)}=1 \text {. }
$$

For instance, if $p_{n}=c / \sqrt{n}(c>0)$, we have $P(n) \sim 2 c \sqrt{n}$ and thus with probability 1

$$
\lim _{k \rightarrow+\infty} \frac{v_{k}}{k^{2}}=\frac{1}{4 c^{2}}
$$

while if $p_{n}=c / n(c>0)$ we have $P(n) \sim c \log n$ and thus

$$
\lim _{k \rightarrow+\infty} \sqrt[k]{v_{k}}=e^{\mathbf{1} / c}
$$

Still more can be said about the sequence $\left\{\nu_{k}\right\}$. As a matter of fact, by the central limit theorem ([8], p. 130-131), putting $V(n)=\sum_{k=1}^{n} p_{k}^{2}$ we
have

$$
\lim _{n \rightarrow+\infty} P\left(\frac{\sum_{k=1}^{n} \xi_{k}-P(n)}{\sqrt{P(n)-V(n)}}<x\right)=\Phi(x)=\frac{1}{\sqrt{2 \pi}} \int_{-\infty}^{x} e^{-u^{2} / 2} d u .
$$

It follows that in the case $p_{n}=c / \sqrt{n}(c>0)$ for instance we have

$$
\lim _{n \rightarrow+\infty} P\left(\frac{v_{k}-k^{2} / 4 c^{2}}{k^{3 / 2} / 2 c^{2}}<x\right)=\Phi(x),
$$

i. e. the fluctuations of $v_{k}$ around $k^{2} / 4 c^{2}$ are of the order $k^{3 / 2}$ and are approximately normally distributed if $k$ is large. 
It may be added that if we change only slightly the probabilities $p_{n}$, all assertions which were true with probability 1 remain true with the same probability. In fact, according to a theorem of Kakutani [1.2], this is true if we replace $p_{n}$ by $p_{n}^{\prime}$ provided that

$$
\sum_{n=1}^{\infty} \frac{\left(p_{n}-p_{n}^{\prime}\right)^{2}}{p_{n}\left(1-p_{n}\right)}<+\infty
$$

Thus in the case of $p_{n}=c / \sqrt{n}$, we may replace $p_{n}$ by $p_{n}^{\prime}=c / \sqrt{n}+d / n^{\alpha}$ with $\alpha>\frac{3}{4}$. We shall see, however, that for our problems still larger changes of the probabilities are admissible if those changes do not affect certain averages.

\$2. Two independent random sequences. Let $\left\{v_{k}\right\}$ and $\left\{\mu_{k}\right\}$ be independent random sequences of positive integers, generated by the sequences $\left\{p_{n}\right\}$ and $\left\{q_{n}\right\}$ of probabilities $\sum_{n=1}^{\infty} p_{n}=\sum_{n=1}^{\infty} q_{n}=+\infty$, i. e. $v_{k}$ and $\mu_{k}$ respectively are the $k$-th values of $n$ for which $\xi_{n}=1$ and respectively $\eta_{n}=1$, where the random variables $\xi_{1}, \eta_{1}, \ldots, \xi_{n}, \eta_{n}, \ldots$ taking on only the values 0 and 1 are completely independent, $P\left(\xi_{n}=1\right)=p_{n}$ and $P\left(\eta_{n}=1\right)=q_{n}$. Let $f(n)$ denote the number of representations of $n$ in the form $n=v_{k}+\mu_{l}$. First we prove

THEOREM 1. If

$$
\lim _{n \rightarrow+\infty} p_{n}=\lim _{n \rightarrow+\infty} q_{n}=0
$$

then $f(n)$ has in the limit for $n \rightarrow+\infty$ a Poisson distribution with mean value $\lambda$, i. e.

$$
\lim _{n \rightarrow+\infty} P(f(n)=r)=\frac{\lambda^{r} e^{-\lambda}}{r !} \quad(r=0,1, \ldots) .
$$

Proof. As the random variables $\xi_{k} \eta_{n-k}(k=1,2 ; \ldots, n-1)$ are by supposition independent and

$$
f(n)=\sum_{k=1}^{n-1} \xi_{k} \eta_{n-k}
$$

(1) Clearly (2.1) and (2.2) imply $\sum_{k=1}^{\infty} p_{k}=\sum_{k=1}^{\infty} q_{k}=+\infty$.
Theorem 1. follows from a well-known general theorem of probability theory (see [8], p. $132-133$ ).

Let $S_{r}$ denote the sequence of those integers $n$ for which $f(n)=r$; Theorem 1 suggests that $S_{r}$ will have (with probability 1 ) a density equal to $\lambda^{r} e^{-\lambda} / r !(r=0,1, \ldots)$. This is in fact true under general conditions, contained in the following

THEOREM 2. If the independent random sequences $\nu_{k}$ and $\mu_{k}$ are generated by the sequences of probabilities $p_{n}$ and $q_{n}$ where $p_{n}$ and $q_{n}$ are decreasing and tend to zero, if further (2.2) is satisfied and there exists a constant $\delta$ with $0<\delta<1$ such that

$$
\sum_{k=1}^{n} p_{k}=O\left(n^{1-\delta}\right) \quad \text { and } \quad \sum_{k=1}^{n} q_{k}=O\left(n^{1-\delta}\right),
$$

then, denoting by $S_{r}$ the set of those positive integers $n$ which have exactly $r$ representations of the form $n=\nu_{k}+\mu_{l}$, we find that $S_{r}$ has with probability 1 the density $\lambda^{r} e^{-\lambda} / r !(r=0,1, \ldots)$

COROLLARY. Under the conditions of Theorem 2 the sequence of all those integers which can be represented in the form $n=\nu_{k}+\mu_{l}$ has with probability 1 the density $1-e^{-\lambda}$.

Proof of Theorem 2. The validity of Theorem 2 is a consequence of the fact that the random variables $f(n)(n=1,2, \ldots)$ are in a certain sense almost independent, as will be seen from the proof given below.

Let $\varepsilon_{r}(n)$ be equal to 1 if $f(n)=r$ and 0 otherwise. Then we have

$$
M\left(\varepsilon_{r}(n)\right)=\sum p_{k_{1}} q_{n-k_{1}} \ldots p_{k_{r}} q_{n-k_{r}} \prod_{j \neq k_{h}(h=1,2, \ldots, r)}\left(1-p_{j} q_{n-j}\right),
$$

where the summation is extended over all $r$-tuples of different integers $\left(k_{1}, \ldots, k_{r}\right)$ such that $1 \leqslant k_{1}<k_{2}<\ldots<k_{r} \leqslant n-1$.

We shall need here and in what follows the following inequalities, valid for any sequence $a_{1}, a_{2}, \ldots, a_{N}$ of positive numbers:

$$
\frac{\left(\sum_{k=1}^{N} a_{k}\right)^{r}}{r !}\left(1-\frac{\left(\sum_{k=1}^{N} a_{k}^{2}\right)\left(\begin{array}{l}
r \\
2
\end{array}\right)}{\left(\sum_{k=1}^{N} a_{k}\right)^{2}}\right) \leqslant \sum_{1 \leqslant k_{1}<k_{2}<\ldots<k_{r} \leqslant N} a_{k_{1}} a_{k_{2}} \ldots a_{k_{r}} \leqslant \frac{\left(\sum_{k=1}^{N} a_{k}\right)^{r}}{r !}
$$

The upper inequality in (2.7) is trivial; the lower inequality, which has already been used in a previous paper ([7], p. 27) of the authors, also easily follows from the polynomial theorem.

It follows from (2.6) by using (2.7), with respect to (2.2) that

$$
\lim _{n \rightarrow+\infty} M\left(\varepsilon_{r}(n)\right)=\frac{\lambda^{r} e^{-\lambda}}{r !} \quad(r=0,1, \ldots) .
$$


Now we consider $M\left(\varepsilon_{r}(n) \varepsilon_{r}(m)\right)$ for $n<m$. Let $A_{k}$ denote the event $\xi_{k}=\eta_{n-k}=\eta_{m-k}=1$ and $B_{k}$ the event $\eta_{k}=\xi_{n-k}=\xi_{m-k}=1$; further put, $A=\sum_{k=1}^{n-1} A_{k}$ and $B=\sum_{k=1}^{n-1} B_{k}$; then we evidently have

$$
\begin{aligned}
M\left(\varepsilon_{r}(n) \varepsilon_{r}(m) \mid \bar{A} \bar{B}\right) P(\bar{A} \bar{B}) & =\sum^{\prime} p_{k_{1}} q_{n-k_{1}} \ldots p_{k_{r}} q_{n-k_{r}} p_{l_{1}} q_{m-l_{1}} \ldots p_{l_{r}} q_{m-l_{r}} \times \\
\times & \prod_{k \neq k_{i}, l \neq l_{i}(i=1,2, \ldots, r)}\left(1-p_{k} q_{n-k}\right)\left(1-p_{l} q_{m-l}\right),
\end{aligned}
$$

where $\Sigma^{\prime}$ denotes that the summation is extended over those pairs of $r$-tuples $1 \leqslant k_{1}<k_{2}<\ldots<k_{r}<n$ and $1 \leqslant l_{1}<l_{2}<\ldots<l_{r}<m$ which are disjoint $\left(k_{i} \neq l_{j}\right)$ and such that the $r$-tuples $\left(n-k_{1}, \ldots, n-k_{r}\right)$ and $\left(m-l_{1}, \ldots, m-l_{r}\right)$ are also disjoint. Thus

$$
M\left(\varepsilon_{r}(n) \varepsilon_{r}(m) \mid \bar{A} \bar{B}\right) P(\bar{A} \bar{B}) \leqslant M\left(\varepsilon_{r}(n)\right) M\left(\varepsilon_{r}(m)\right) .
$$

Now clearly $\bar{A} \cdot \bar{B}=\overline{A+B}$ and thus

(2.10) $\quad M\left(\varepsilon_{r}(n) \varepsilon_{r}(m)\right)$

$$
=M\left(\varepsilon_{r}(n) \varepsilon_{r}(m) \mid A+B\right) P(A+B)+M\left(\varepsilon_{r}(n) \varepsilon_{r}(m) \mid \bar{A} \cdot \bar{B}\right) P(\bar{A} \bar{B})
$$

and as $\varepsilon_{r}(n) \varepsilon_{r}(m) \leqslant 1$, we have

(2.11) $\quad M\left(\varepsilon_{r}(n) \varepsilon_{r}(m) \mid A+B\right) P(A+B) \leqslant P(A+B) \leqslant \sum_{k=1}^{n-1}\left(P\left(A_{k}\right)+P\left(B_{k}\right)\right)$.

Thus from (2.9), (2.10) and (2.11) it follows that

(2.12) $M\left(\varepsilon_{r}(n) \varepsilon_{r}(m)\right)-M\left(\varepsilon_{r}(n)\right) M\left(\varepsilon_{r}(m)\right) \leqslant \sum_{k=1}^{n-1}\left(p_{k} \dot{q}_{n-k}^{\prime} q_{m-k}+q_{k} p_{n-k} p_{m-k}\right)$ and thus

(2.13) $\quad M\left(\varepsilon_{\boldsymbol{r}}(n) \varepsilon_{\boldsymbol{r}}(m)\right)-M\left(\varepsilon_{\boldsymbol{r}}(n)\right) M\left(\varepsilon_{\boldsymbol{r}}(m)\right)$

$$
\leqslant\left(q_{m-n}+p_{m-n}\right) \sum_{k=1}^{n-1} p_{k} q_{n-k} \leqslant C_{1}\left(q_{m-n}+p_{m-n}\right) .
$$

Let us denote by $\zeta_{r}(N)$ the number of those values of $n \leqslant N$ for which $f(n)=r$, i. e. put

$$
\zeta_{r}(N)=\frac{1}{N} \sum_{n=1}^{N} \varepsilon_{r}(n)
$$

It follows from (2.8) that

$$
\lim _{N \rightarrow+\infty} M\left(\zeta_{r}(N)\right)=\frac{\lambda^{r} e^{-\lambda}}{r !}
$$

further, by (2.13) and by our supposition (2.5) we have

$$
D^{2}\left(\zeta_{r}(N)\right) \leqslant C_{2} \sum_{k=1}^{N-1}\left(p_{k}+q_{k}\right) \frac{(N-k)}{N^{2}}=O\left(\frac{1}{N^{\delta}}\right) .
$$

Thus by the inequality of Chebyshev (see [9]) we obtain for $\varepsilon>0$

$$
P\left(\left|\zeta_{r}(N)-M\left(\zeta_{r}(N)\right)\right|>\varepsilon\right)=O\left(\frac{1}{N^{\delta}}\right) .
$$

Thus the series

$$
\sum_{j=1}^{\infty} P\left(\left|\zeta_{r}\left(N_{j}\right)-M\left(\zeta_{r}\left(N_{j}\right)\right)\right|>\varepsilon\right)
$$

is convergent if $N_{j}=\left[j^{2 / \delta}\right]$ for any $\varepsilon>0$ and thus by the Lemma of BorelCantelli and by (2.15) we have with probability 1

$$
\lim _{j \rightarrow+\infty} \zeta_{r}\left(N_{j}\right)=\frac{\lambda^{r} e^{-\lambda}}{r !} \quad(r=0,1, \ldots) .
$$

Since evidently for $N_{j}<N<N_{j+1}$

$$
\zeta_{r}\left(N_{j}\right) \frac{N_{j}}{N_{j+1}} \leqslant \zeta_{r}(N) \leqslant \zeta_{r}\left(N_{j+1}\right) \frac{N_{j+1}}{N_{j}}
$$

and clearly $\lim _{j \rightarrow+\infty} \frac{N_{j_{+1}}}{N_{j}}=1$, it follows from (2.19) that with probability 1

$$
\lim _{N \rightarrow+\infty} \zeta_{r}(N)=\frac{\lambda^{r} e^{-\lambda}}{r !} \quad(r=0,1, \ldots)
$$

Thus Theorem 2 is proved. The Corollary is an evident consequence of the assertion of Theorem 2 for $r=0$, since the sequence of those numbers $n$ which have at least one representation in the form $n=\nu_{k}+\mu_{l}$ is the complementary set of the sequence $S_{0}$.

Clearly if $p_{n}=a / \sqrt{n}$ and $q_{n}=b / \sqrt{n}$ with $a>0, b>0$ then

$$
\sum_{k=1}^{n-1} p_{k} q_{n-k}=a b \sum_{k=1}^{n-1} \frac{1}{\sqrt{k(n-k)}}=a b \int_{0}^{1} \frac{d x}{\sqrt{x(1-x)}}+O\left(\frac{1}{\sqrt{n}}\right)=a b \pi+o\left(\frac{1}{\sqrt{n}}\right)
$$


and finally

$$
\sum_{k=1}^{n-1} p_{k}=O\left(n^{1 / 2}\right), \quad \sum_{k=1}^{n-1} q_{k}=O\left(n^{1 / 2}\right)
$$

thus the conditions of Theorem 2 are fulfilled with $\lambda=a b \pi$ and $\delta=\frac{1}{2}$.

\$3. A square and a random integer. In this section we consider the sums $k^{2}+\mu_{l}$ where $\left\{\mu_{l}\right\}$ is a random sequence generated by the sequence of probabilities $\left\{q_{n}\right\}$. For the sake of simplicity we restrict ourselves to the special case $q_{n}=c / \sqrt{n}(c>0)$, though our result could be proved also under more general suppositions.

THeOREM 3. If $P\left(\eta_{n}=1\right)=q_{n}=c / \sqrt{n}$ with $c>0$ and $f(n)$ denotes the number of representations of $n$ in the form $n=k^{2}+\mu_{l}$, then, if we denote by $S_{r}$ the sequence of integers $n$ for which $f(n)=r, S_{r}$ has with probability 1 the density $\lambda^{r} e^{-\lambda} / r$ ! where $\lambda=c \pi / 2 \quad(r=0,1, \ldots)$.

Proof. The proof follows the same pattern as that of Theorem 2. Let $\varepsilon_{r}(n)$ be equal to 1 if $f(n)=r$ and 0 otherwise. Then we have

$$
M\left(\varepsilon_{r}(n)\right)=\sum \frac{e^{r}}{\sqrt{\left(n-j_{1}^{2}\right) \ldots\left(n-j_{r}^{2}\right)}} \prod_{j \neq j_{h}(h=1,2, \ldots, r)}\left(1-\frac{c}{\sqrt{n-j^{2}}}\right),
$$

where the summation is extended over all $r$-tuples of different integers $j_{1}, j_{2}, \ldots, j_{r}$ with $j_{h}^{2}<n$. Now clearly if $n=N^{2}+h(h=1,2, \ldots, 2 N+1)$, then

$$
\sum_{1 \leqslant j^{2}<n} \frac{1}{\sqrt{n-j^{2}}}=\int_{0}^{1} \frac{d x}{\sqrt{1-x^{2}}}+\frac{1}{\sqrt{h}}+O\left(\frac{1}{\sqrt{n}}\right)
$$

therefore

$$
M\left(\varepsilon_{r}(n)\right)=\frac{\left(\frac{\pi c}{2}+\frac{c}{\sqrt{h}}+O\left(\frac{1}{\sqrt{n}}\right)\right)^{r}}{r !} \exp \left\{-\left(\frac{\pi c}{2}+\frac{c}{\sqrt{h}}+O\left(\frac{1}{\sqrt{n}}\right)\right)\right\}
$$

and thus

$$
\frac{1}{N} \sum_{n=1}^{N} M\left(\varepsilon_{r}(n)\right)=\frac{(\pi c / 2)^{r} e^{-\pi c / 2}}{r !}\left(1+O\left(\frac{1}{\sqrt[4]{N}}\right)\right)
$$

Now let us find for $n<m$ the mean value of $\varepsilon_{r}(n) \varepsilon_{r}(m)$. Let $H_{N}$ denote the set of integers $N-1, N-4, \ldots, N-h^{2}, \ldots, N-[\sqrt{N}]^{2}$. Then the intersection of the sets $H_{n}$ and $H_{m}$, which we denote by $H_{n} H_{m}$, does not contain more than $d(m-n)$ elements, where $d(N)$ denotes the number of divisors of $N$. As a matter of fact, each $l \in H_{n} H_{m}$ is of the form $l=n-j^{2}$ $=m-h^{2}$, i. e. corresponds to a representation of $m-n$ in the form $m-n$ $=h^{2}-j^{2}=(h-j)(h+j)$. Now every decomposition of $m-n$ into the product of two of its divisors of the same parity $m-n=d_{1} d_{2}$ corresponds to exactly one number $l \in H_{n} H_{m}$, namely to $l=n-j^{2}=m-h^{2}$ with $h=\left(d_{1}+d_{2}\right) / 2, j=\left(d_{1}-d_{2}\right) / 2$, and thus our assertion is proved.

Now let $A_{n m}$ denote the event that $\xi_{l}=0$ for all $l \in H_{n m}$. Then we have

$$
P\left(A_{n m}\right)=\prod_{l \in H_{n m}}\left(1-p_{l}\right)=\prod_{h=\left(d_{1}+d_{2}\right) / 2, \vec{d}_{1} d_{2}=m-n}\left(1-\frac{c}{\sqrt{m-h^{2}}}\right) .
$$

We distinguish two cases. Let $E$ denote the class of integers $N$ for which $N$ is contained in an interval $s^{2}-\sqrt[3]{s}, s^{2}+\sqrt[3]{s}(s=2,3, \ldots)$. If $N$ is not in $E$, we have by $(3.5)$

$$
P\left(A_{n m}\right)=1-O\left(\frac{d(m-n)}{\sqrt[12]{m}}\right)=1-O\left(\frac{1}{m^{1 / 13}}\right)
$$

if $m \geqslant m_{0}$, in view of $d(N)=O\left(N^{\varepsilon}\right)$ for every $\varepsilon>0$ (see [15], p. 260). Evidently under the condition $A_{n m}$ the random variables $\varepsilon_{r}(n)$ and $\varepsilon_{r}(m)$ are independent; thus if $m$ is not in $E$, we have

$$
\begin{aligned}
& M\left(\varepsilon_{r}(m) \mid A_{n m}\right)=M\left(\varepsilon_{r}(m)\right)+O\left(\frac{1}{m^{1 / 13}}\right), \\
& M\left(\varepsilon_{r}(n) \mid A_{n m}\right)=M\left(\varepsilon_{r}(n)\right)+O\left(\frac{1}{m^{1 / 13}}\right)
\end{aligned}
$$

and

$$
\begin{aligned}
M\left(\varepsilon_{r}(m) \varepsilon_{\boldsymbol{r}}(n)\right) & =M\left(\varepsilon_{\boldsymbol{r}}(m) \varepsilon_{\boldsymbol{r}}(n) \mid A_{n m}\right)+O\left(\frac{1}{m^{1 / 13}}\right) \\
& =M\left(\varepsilon_{\boldsymbol{r}}(m)\right) M\left(\varepsilon_{r}(n)\right)+O\left(\frac{1}{m^{1 / 13}}\right)
\end{aligned}
$$

It follows that

(3.7) $\sum_{n=1}^{N} \sum_{m=1}^{N}\left[M\left(\varepsilon_{r}(m) \varepsilon_{r}(n)\right)-M\left(\varepsilon_{r}(m)\right) M\left(\varepsilon_{r}(n)\right)\right]$

$$
=O\left(N^{2-1 / 13}\right)+O\left(N \sum_{m_{\epsilon}, m \leqslant N} 1\right) .
$$


Since

$$
\sum_{m \in E, m \leqslant N} 1=O\left(N^{5 / 6}\right)
$$

it follows that

$$
\sum_{n=1}^{N} \sum_{m=1}^{N}\left[M\left(\varepsilon_{r}(n) \varepsilon_{r}(m)\right)-M\left(\varepsilon_{r}(n)\right) M\left(\varepsilon_{r}(m)\right)\right]=O\left(N^{25 / 13}\right) .
$$

Thus putting

$$
\zeta_{r}(N)=\frac{1}{N} \sum_{n=1}^{N} \varepsilon_{r}(n)
$$

we have

$$
M\left(\zeta_{r}(N)\right)=\frac{\lambda^{r} e^{-\lambda}}{r !}+O\left(\frac{1}{\sqrt[4]{N}}\right)
$$

and

$$
D^{2}\left(\zeta_{r}(N)\right)=O\left(\frac{1}{N^{1 / 13}}\right)
$$

By the inequality of Chebyshev we obtain for any $\varepsilon>0$

$$
P\left(\left|\zeta_{r}(N)-\frac{\lambda^{r} e^{-\lambda}}{r !}\right|>\varepsilon\right)=O\left(\frac{1}{N^{1 / 13}}\right) .
$$

Thus the series

$$
\sum_{N=1}^{\infty} \dot{P}\left(\left|\zeta_{r}\left(N^{14}\right)-\frac{\lambda^{r} e^{-\lambda}}{r !}\right|>\varepsilon\right)
$$

is convergent.

Since this holds for any $\varepsilon>0$, it follows by the Borel-Cantelli lemma that with probability 1

$$
\lim _{N \rightarrow+\infty} \zeta_{r}\left(N^{14}\right)=\frac{\lambda^{r} e^{-\lambda}}{r !} \quad(r=0,1, \ldots)
$$

Since

$$
\lim _{N \rightarrow+\infty}\left(\frac{N+1}{N}\right)^{14}=1
$$

it follows, as in the proof of Theorem 2, that with probability 1

$$
\lim _{N \rightarrow+\infty} \zeta_{r}(N)=\frac{\lambda^{r} e^{-\lambda}}{r !} \quad(r=0,1, \ldots),
$$

which proves Theorem 3 .
§ 4. Sum of two terms of the same random sequence. In this section we prove

THEOREM 4. Let $v_{k}$ be a random sequence of positive integers generated by the sequence of probabilities $p_{n}$ such that $p_{n}$ is decreasing and tends to 0 ; further, let

$$
\lim _{n \rightarrow+\infty} \sum_{k<n / 2} p_{k} p_{n-k}=\lambda
$$

and for some $\delta$ with $0<\delta<1$

$$
\sum_{k=1}^{n} p_{k}=O\left(n^{1-\delta}\right)
$$

Let $f(n)$ denote the number of representations of $n$ in the form $n=v_{k}+v_{l}$ with $v_{k}<v_{l}$, and denote by $S_{r}$ the sequence of those integers $n$ for which $f(n)=r$. Then $S_{r}$ has with probability 1 the density $\lambda^{r} e^{-\lambda} / r !$ for $r=0,1, \ldots$ Especially the set of those integers $n$ which have at least one representation in the form $n=v_{k}+v_{l}$ with $k<l$ has the density $1-e^{-\lambda}$.

The proof of Theorem 4 follows exactly the same pattern as that of Theorem 2 and thus may be left to the reader. Clearly if $p_{n}=a / \sqrt{n}$ with $a>0$ then the conditions of Theorem 4 are fulfilled with $\lambda=\pi a^{2} / 2$ and $\delta=\frac{1}{2}$, since

$$
\sum_{k<n / 2} p_{k} p_{n-k}=a^{2} \int_{0}^{1 / 2} \frac{d x}{\sqrt{x(1-x)}}+O\left(\frac{1}{\sqrt{n}}\right)=\frac{a^{2} \pi}{2}+O\left(\frac{1}{\sqrt{n}}\right)
$$

further

$$
\sum_{k \leq n_{i} 2} p_{k}=O(\sqrt{n})
$$

Let us mention that if we define $f^{*}(n)$ as the number of all representations of $n$ in the form $n=v_{k}+v_{l}$ without the restriction $v_{k}<v_{l}$, then clearly $f^{*}(n)=2 f(n)$ if $n$ is odd and $f^{*}(n)=2 f(n)+\xi_{n / 2}$ if $n$ is even.

Clearly the sequence of those even numbers $2 k$ for which $\xi_{k}=1$ has the density 0 , as a matter of fact, if $N \eta_{N}$ denotes the number of such integers $2 k \leqslant 2 N$, we have

$$
\eta_{N}=\frac{1}{N} \sum_{k=1}^{N} \xi_{k}
$$

and thus

$$
M\left(\eta_{N}\right)=O\left(\frac{1}{\sqrt{N}}\right)
$$


This implies

$$
\sum M\left(\eta_{N^{4}}\right)<+\infty
$$

therefore with probability 1

$$
\lim _{N \rightarrow+\infty} \eta_{N^{4}}=0
$$

and thus also

$$
\lim _{n \rightarrow+\infty} \eta_{n}=0 \text {. }
$$

Thus it follows from Theorem 4 that if $S_{r}^{*}$ denotes the sequence of those integers $n$ for which $f^{*}(n)=r$, then $S_{2 r}^{*}$ has with probability 1 . the density $\lambda^{r} e^{-\lambda} / r$ ! while $S_{2 r+1}^{*}$ has the density $0(r=0,1, \ldots)$.

§5. On random sequences for which $f(n) \rightarrow+\infty$. Let us choose a sequence $\left\{p_{n}\right\}$ of probabilities so that putting

$$
A_{1}(n)=\sum_{k<n / 2} p_{k} p_{n-k}
$$

and

we shall have

$$
A_{2}(n)=\sum_{k \leq n / 2} p_{k}^{2} p_{n-k}^{2}
$$

$$
\lim _{n \rightarrow+\infty}\left(A_{1}(n)-A_{2}(n)\right)=+\infty .
$$

Let $v_{l c}$ denote the random sequence of integers generated by the sequence $p_{n}$ in the sense of $\S 1$. In that case the number of representations of $n$ in the form $n=v_{k}+v_{l}$ with $k<l$ is in the limit for $n \rightarrow+\infty$ normally distributed. This is expressed by the following

THEOREM 5. Let $\left\{p_{n}\right\}$ be a sequence of probabilities such that if $A_{1}(n)$ and $A_{2}(n)$ are defined by (5.1) and (5.2) respectively, then we have (5.3). Then, denoting by $f(n)$ the number of representations of $n$ in the form $n=v_{l k}+v_{l}$ with $k<l$, we have for $-\infty<x<+\infty$

$$
\lim _{n \rightarrow+\infty} P\left(\frac{f(n)-A_{1}(n)}{\sqrt{A_{1}(n)-A_{2}(n)}}<\infty\right)=\Phi(x),
$$

where $\Phi(x)$ denotes the standard form of the normal distribution function, i.e.

$$
\Phi(x)=\frac{1}{\sqrt{2 \pi}} \int_{-\infty}^{x} e^{-u^{2} / 2} d u
$$

Proof. $f(n)=\sum_{k<n / 2} \xi_{k} \xi_{n-k}$, where the random variables $\xi_{k}$ are independent and $P\left(\xi_{k}=1\right)=p_{k}, \quad P\left(\xi_{k}=0\right)=1-p_{k} \quad(k=1,2, \ldots)$. It follows that

$$
M(f(n))=A_{1}(n) \quad \text { and } \quad D^{2}(f(n))=A_{1}(n)-A_{2}(n) .
$$

Since the conditions of the central limit theorem of probability theory (e. g. Lindeberg's conditions, see [8]) are fulfilled, (5.4) follows immediately.

The conditions of Theorem 5 are clearly fulfilled, e. g. if $p_{n}=\omega(n) / \sqrt{n}$ where $\omega(n) \rightarrow+\infty$ and $\omega(n) / \sqrt{n} \leqslant q<1$.

A similar result holds for the number of representations of $n$ in the form $n=k^{2}+\mu_{l}$ where $\left\{\mu_{l}\right\}$ is a random sequence of integers generated by a sequence of probabilities $q_{n}$ such that putting $A_{1}(n)=\sum_{k^{2}<n} q_{n-k^{2}}$ and $A_{2}(n)=\sum_{k^{2}<n} q_{n-k^{2}}^{2}$ we have $A_{1}(n)-A_{2}(n) \rightarrow+\infty$; thus for instance if $q_{n}$ $=\omega(n) / \sqrt{n}$ with $\omega(n) \rightarrow+\infty$ and $\omega(n) / \sqrt{n} \leqslant q<1$. In this case if we denote by $f(n)$ the number of representations of the number $n$ in the form $n=k^{2}+v_{l},(5.4)$ is valid.

§6. Sum of more than two terms of a random sequence. The results obtained in the preceding sections can be generalized to sums of more than two terms. As an example we prove the following

THEOREM 6. Let $\boldsymbol{y}_{k}$ be a random sequence of integers generated by the sequence of probabilities $p_{n}=c / n^{1-1 / s}$ where $s \geqslant 3$ is a positive integer and $c>0$. Let $f(n)$ denote the number of representations of the number $n$ in the form $n=v_{k_{1}}+v_{k_{2}}+\ldots+v_{k_{s}}$ with $k_{1}<k_{2}<\ldots<k_{s}$. Let $S_{r}$ denote the seform $n=v_{k_{1}}+v_{k_{2}}+\ldots+v_{k_{s}}$ with $k_{1}<h_{2}<\ldots$ of those integers $n$ for which $f(n)=r$. Then $S_{r}$ has with probability 1 the density $\lambda^{r} e^{-\lambda} / r$ ! where $\lambda=\frac{e^{s} \Gamma(1 / s)^{s}}{s !}(r=0,1, \ldots)$.

Proof. Let us put $\varepsilon_{r}(n)=1$ if $f(n)=r$ and $=0$ otherwise. Now we have

$$
\begin{aligned}
& \text { (6.1) } \lim _{n \rightarrow+\infty} \sum_{\substack{k_{1}<k_{2}<\ldots<k_{s} \\
k_{1}+k_{2}+\ldots+k_{s}=n}} p_{k_{1}} p_{k_{2}} \ldots p_{k_{s}} \\
& =\frac{c^{s}}{s !} \int_{\substack{\left.0 \leq x_{i}<1 \\
x_{1}+x_{2}+\ldots+x_{s-1}<1, \ldots, s-1\right)}} \ldots \int_{\substack{i=1,2 \\
x_{1}}} \frac{d x_{1}}{x_{1}^{1-1 / s}} \cdot \frac{d x_{2}}{x_{2}^{1-1 / s}} \ldots \frac{d x_{s-2}}{x_{s-2}^{1-1 / s}} \cdot \frac{d x_{s-1}}{x_{s-1}^{1-1 / s}\left(1-x_{1}-\ldots-x_{s-1}\right)^{1-1 / s}}
\end{aligned}
$$

and the integral on the right of (6.1) is equal to $\Gamma(1 / s)^{s}$. Thus putting

$$
\lambda=\frac{(c \Gamma(1 / s))^{s}}{s !},
$$


we hàve

$$
\lim _{n \rightarrow+\infty} M\left(\varepsilon_{r}(n)\right)=\frac{\lambda^{r} e^{-\lambda}}{r !} \quad(r=0,1, \ldots) .
$$

The estimation of $M\left(\varepsilon_{\boldsymbol{r}}(n) \varepsilon_{\boldsymbol{r}}(m)\right)$ can be obtained as before, and Theorem 6 is proved in the same way as Theorems 2-4.

\$ 7. Differences formed from a random sequence. Let $\left\{v_{k}\right\}$ be a random sequence generated by the sequence of probabilities $\left\{p_{n}\right\}$. Let $g(n)$ denote the number of representations of $n$ in the form $n=v_{k}-v_{l}$. Clearly

$$
M(g(n))=\sum_{k=1}^{n} p_{k} p_{n+k} .
$$

Two cases are possible: either $M(g(n))$ is infinite, which is of minor interest, or it is finite for every $n$. The latter is the case if for instance $\sum_{k=1}^{\infty} p_{k}^{2}<+\infty$. In that case, however, we have $\lim _{n \rightarrow+\infty} M(g(n))=0$. Hence it is clear that here we cannot hope to obtain similar results to those we obtained for the sums $v_{k}+v_{l}$. More reasonable results can be obtained if we consider only representations $n=v_{k}-v_{l}$ for which $v_{k} \leqslant(1+B) n$ where $B$ is a positive constant not depending on $n$. If $g_{B}(n)$ denotes the number of such representations of $n$, we have, putting $p_{k}=c / \sqrt{k}(c>0)$,

$$
\lim _{n \rightarrow+\infty} M\left(g_{B}(n)\right)=c^{2} \int_{0}^{B} \frac{d x}{\sqrt{x(1+x)}}=c^{2} \log \left(B+1+\sqrt{B^{2}+2 B}\right) .
$$

It can be shown by the same method as that used in proving Theorem 2 that the following theorem holds:

THEOREM 7. Let the random sequence $v_{k}$ be generated by the sequence of probabilities $p_{n}=c / \sqrt{n}(c>0)$; let $g_{B}(n)$ denote the number of representations of $n$ of the form $n=v_{k}-v_{l}$ where $\nu_{k} \leqslant(1+B) n(B>0)$; then the sequence of those integers $n$ for which $g_{B}(n)=r$ has the density $\lambda^{r} e^{-\lambda} / r$ !, where $\lambda=c^{2} \log \left(B+1+\sqrt{B^{2}+2 B}\right)$. Especially for $B=(e-1)^{2} / 2 e$ we have $\lambda=c^{2}$.

§ 8. On random sequences for which $f(n)$ is bounded. S. Sidon ([7], [12]) considerf $d$ such sequences $\left\{a_{k}\right\}$ of positive integers that the sequence of coefficients of the series $\left(\sum_{k=1}^{\infty} x^{a_{k}}\right)^{l}$ is bounded. He called such sequences $B_{l}$-sequences $(l=2,3, \ldots)$. We shall call a sequence $\left\{a_{k}\right\}$ a $B_{l}(K)$ sequence if the coefficients of $\left(\sum_{k=1}^{\infty} x^{a_{k}}\right)^{l}$ are all $\leqslant K$. The interesting ques- tion is how dense a $B_{l}(K)$ sequence can be. It has been shown by Erdös [3] that there exists a $B_{2}(1)$ sequence $\left\{a_{k}\right\}$ such that $a_{k}<c k^{3}$, while Erdös and Turán [11] (for a detailed proof see [13]) have proved that. for every $B_{2}(1)$ sequence one has $\limsup _{k \rightarrow+\infty} a_{k} / k^{2}=+\infty$.

We shall prove in this section that if $\left\{v_{k}\right\}$ is a random sequence generated by the sequence of probabilities $p_{n}=c / n^{1 / 2+8}(c>0, \varepsilon>0)$ then with probability $1, v_{k}$ is a $B_{2}$ sequence.

Thus we shall prove the following

THEOREM 8. Let $v_{k}$ be a random sequence of positive integers generated by the sequence of probabilities $p_{n}=c / n^{1 / 2+\varepsilon}$ where $c>0$ and $0<\varepsilon<\frac{1}{2}$. Let $f(n)$ denote the number of representations of $n$ in the form $n=v_{k}+v_{l}$ with $k \leqslant l$; then with probability $1 f(n)$ is bounded; moreover with probability $1 f(n) \leqslant[1 / 2 \varepsilon]$ except perhaps for a finite number of values of $n$.

Pro of. Let $\xi_{n}$ have the same meaning as in $\S 1$. Then $f(n)=\sum_{k \leqslant n / 2} \xi_{k} \xi_{n-k}$ and thus, taking into account that

$$
\lim _{n \rightarrow+\infty} n^{2 \varepsilon}\left(\sum_{k \leqslant n / 2} p_{k} p_{n-k}\right)=c^{2} \int_{0}^{1 / 2} \frac{d x}{[x(1-x)]^{1 / 2+\varepsilon}}=\frac{1}{2} c^{2} I(\varepsilon),
$$

we have

$$
M\left(e^{l f(n)}\right)=\prod_{k \leqslant n / 2}\left(1+\frac{\left(e^{t}-1\right) c^{2}}{(k(n-k))^{1 / 2+\varepsilon}}\right) \leqslant \exp \frac{c^{2}\left(e^{t}-1\right) I(\varepsilon)}{n^{2 s}}
$$

for $n \geqslant n_{0}$ where

$$
I(\varepsilon)=B\left(\frac{1}{2}-\varepsilon, \frac{1}{2}-\varepsilon\right)=\frac{\Gamma\left(\frac{1}{2}-\varepsilon\right)^{2}}{\Gamma(1-2 \varepsilon)} .
$$

Here and in what follows $B(\alpha, \beta)$ denotes the beta-function of Euler

$$
B(\alpha, \beta)=\int_{0}^{1} x^{\alpha-1}(1-x)^{\beta-1} d x=\frac{\Gamma(\alpha) \Gamma(\beta)}{\Gamma(\alpha+\beta)} \quad \text { for } \quad \alpha>0, \beta>0 .
$$

We infer, choosing $t=\log \left(1+n^{2 \varepsilon}\right)$, that

$$
P(f(n) \geqslant K+1) \leqslant \frac{\epsilon^{c^{2} I(\varepsilon)}}{n^{2 \varepsilon(K+1)}} .
$$

Thus if $K+1>1 / 2 \varepsilon$ the series

$$
\sum_{n=1}^{\infty} P(f(n) \geqslant K+1)
$$

A.cta Arithmetica VI. 
is convergent. It follows from the Lemma of Borel-Cantelli that with probability $1 f(n) \leqslant K$ except for a finite number of values of $n$; we may choose $K=[1 / 2 \varepsilon]$. Thus Theorem 8 is proved.

Note that from (1.6) we have $\nu_{k} \sim(k / c)^{2 /(1-2 \varepsilon)}$. Thus for any $\delta>0$ there exist sequences $\left\{a_{k}\right\}$ such that $a_{k}=O\left(k^{2+\delta}\right)$ and the number $f(n)$ of representations of $n$ in the form $n=a_{j}+a_{k}$ is bounded. As a matter of fact, if we choose $p_{k}=1 /\left(k^{1 / 2+8}\right)$ with $\varepsilon=\delta /(4+2 \delta)$, almost all realizations of the corresponding random sequence $\left\{v_{k}\right\}$ may be taken for $\left\{a_{k}\right\}$. Of course (as is usual if a number-theoretical statement of existence is proved by probabilistic methods) our methods do not give an effective construction.

By the same method one can prove the existence of $B_{l}$-sequences $\left\{a_{k}\right\}$ such that $a_{k}=O\left(k^{l+\delta}\right)$ for any $\delta>0(l=3,4, \ldots)$.

\section{\$ 9. A theorem of the ,iterated logarithm" type.}

THEOREM 9. If the random sequence $\left\{v_{k}\right\}$ is generated by the sequence of probabilities $p_{n}=c / \sqrt{n}(c>0)$ and $f(n)$ denotes the number of solutions of $n=v_{k}+v_{l}(k<l)$, we have with probability 1

$$
\limsup _{n \rightarrow+\infty} \frac{f(n) \log \log n}{\log n}=1 \text {. }
$$

Pro of. First we prove that with probability 1

$$
\limsup _{n \rightarrow+\infty} \frac{f(n) \log \log n}{\log n} \leqslant 1 \text {. }
$$

Clearly for any $\varepsilon>0$ if $n>n_{0}$ and $R=\left[(1+2 \varepsilon) \frac{\log n}{\log \log n}\right]$

$$
P(f(n) \geqslant R) \leqslant \sum p_{k_{1}} p_{n-k_{1}} \ldots p_{k_{R}} p_{n-k_{R}} \leqslant \frac{\left(\sum_{k<n / 2} c^{2} / \sqrt{k(n-k)}\right)}{R !}
$$

and thus

$$
P\left(f(n)>(1+2 \varepsilon) \frac{\log n}{\log \log n}\right)=O\left(\frac{1}{n^{1+\varepsilon}}\right) .
$$

It follows that the series $\sum P\left(f(n)>(1+2 \varepsilon) \frac{\log n}{\log \log n}\right)$ is convergent for any $\varepsilon>0$, and thus by the Borel-Cantelli Lemma it follows that (9.2) holds.

Now we prove that with probability. 1 ,

$$
\limsup _{n \rightarrow+\infty} \frac{f(n) \log \log n}{\log n} \geqslant 1 \text {. }
$$

To prove (9.4) we need a lower estimate for $P(f(n) \geqslant R)$; we have by (2.7) for $R=\left[(1-2 \varepsilon) \frac{\log n}{\log \log n}\right]+1$ and $n \geqslant n_{0}(\varepsilon)$

$$
P(f(n) \geqslant R) \geqslant \frac{1}{n^{1-\varepsilon}}
$$

which implies that the series $\sum P\left(f(n) \geqslant(1-2 \varepsilon) \frac{\log n}{\log \log n}\right)$ is divergent.

Now let $A_{n}$ denote the event $f(n) \geqslant(1-2 \varepsilon) \frac{\log n}{\log \log n}$; the events $A_{*}$ (while not independent) are almost pairwise independent in the following sense:

$$
\lim _{N \rightarrow+\infty} \frac{\sum_{n=1}^{N} \sum_{m=1}^{N} P\left(A_{n} A_{m}\right)}{\left(\sum_{n=1}^{N} P\left(A_{n}\right)\right)^{2}}=1 .
$$

As we have shown in a previous paper [16], this is sufficient for the validity of the Borel-Cantelli lemma and thus (9.6) and (9.5) imply (9.4). That (9.6) holds can be shown by the same method as that applied in the proof of Theorem 2 in estimating $M\left(\varepsilon_{r}(n) \varepsilon_{r}(m)\right)-M\left(\varepsilon_{r}(n)\right) M\left(\varepsilon_{r}(m)\right)$.

$\S 10$. The law of large numbers for $f(n)$. In this section we prove

THEOREM 10. If the random sequence $\left\{v_{k}\right\}$ is generated by the sequence of probabilities $p_{n}=c / \sqrt{n}(c>0)$ and $f(n)$ denotes the number of solutions of $n=v_{k}+\nu_{l}$ with $k<l$ then with probability 1

$$
\lim _{N \rightarrow+\infty} \frac{1}{N} \sum_{n=1}^{N} f(n)=\lambda
$$

where $\lambda=c^{2} \pi / 2$.

Pro of. Putting

$$
\zeta_{N}=\frac{1}{N} \sum_{n=1}^{N} f(n)
$$

and in view of

$$
\sum_{k=1}^{N} 1 / \sqrt{k}=2 \sqrt{n}+O(1)
$$

we evidently have

$$
M\left(\zeta_{N}\right)=c^{2} \int_{0}^{1} \sqrt{\frac{1-x}{x}} d x+O\left(\frac{1}{\sqrt{N}}\right) .
$$


Since

$$
\int_{0}^{1} \sqrt{\frac{1-x}{x}} d x=B\left(\frac{1}{2}, \frac{3}{2}\right)=\frac{\pi}{2},
$$

where $B(\alpha, \beta)$ is Euler's beta function (see (8.3)), it follows that

$$
M\left(\zeta_{N}\right)=\frac{\pi c^{2}}{2}+O\left(\frac{1}{\sqrt{N}}\right) .
$$

On the other hand, we obtain by some easy calculations

$$
D^{2}\left(\zeta_{N}\right)=\frac{10 c^{3}}{3 \sqrt{N}}+O\left(\frac{1}{N}\right)
$$

Thus by the inequality of Chebysher

$$
P\left(\left|\zeta_{N}-\lambda\right|>\varepsilon\right)=O\left(\frac{1}{\sqrt{N}}\right) .
$$

It follows that the series

$$
\sum_{N=1}^{\infty} P\left(\left|\zeta_{N^{3}}-\lambda\right|>\varepsilon\right)
$$

is convergent for every $\varepsilon>0$; by the Lemma of Borel-Cantelli this implies that with probability 1

$$
\lim _{N \rightarrow+\infty} \zeta_{N^{3}}=\lambda
$$

On the other hand, we have for $A<B<3 A / 2$

$$
M\left(\sum_{n=A+1}^{B} f(n)\right) \sim(B-A) \lambda
$$

and

$$
D^{2}\left(\sum_{n=A+1}^{B} f(n)\right)=O\left(\frac{(B-A)^{2}}{\sqrt{A}}\right)
$$

Thus we have

$$
P\left(\underset{N^{3}<M \leqslant(N+1)^{3}}{\operatorname{Max}}\left|\sum_{N^{3}+1}^{M} f(n)\right|>\varepsilon N^{3}\right)=O\left(\frac{1}{N^{3 / 2}}\right),
$$

and therefore for any $\varepsilon>0$

$$
\sum_{N=1}^{\infty} P\left(\operatorname{Max}_{N^{3}<M \leqslant(N+1)^{3}}\left|\sum_{N^{3}+1}^{M} f(n)\right|>\varepsilon\right)<+\infty .
$$

(10.4) and (10.6) imply that with probability 1

$$
\lim _{N \rightarrow+\infty} \zeta_{N}=\lambda
$$

Thus our Theorem is proved.

To obtain the limiting distribution of $\left(\sum_{n=1}^{N} f(n)-\lambda N\right) / N^{3 / 4}$ the higher moments have to be calculated. We do not investigate this question here.

§ 11. Stochastic analogons of Romanoff's theorem. N. P. Romanoff proves [5] that if $p$ denotes primes and $a>1$ an integer, then the set of integers $n$ which can be represented in the form $n=a^{k}+p(k=1,2, \ldots)$ has positive density. Erdös [14] has shown that if $Q(x)$ is a polynomial, then the sequence of numbers $n$ which can be written in the form $n=Q\left(a^{k}\right)+p$ also has positive density. In this section we shall consider stochastic analogons of these theorems. First we replace the sequence of primes by a random sequence $\left\{\mu_{l}\right\}$ generated by the sequence of probabilities $q_{n}=b / \log (n+1)(b>0)$, in which case by (1.6) we have with probability $1 \mu_{l} \sim b l \log l$, i. e. for $b=1$ the sequence $\mu_{l}$ has the same order of magnitude as the $l$-th prime number.

We prove the following

THEOREM 11. Let the random sequence $\left\{\mu_{l}\right\}$ be generated by the sequence of probabilities $q_{n}=b / \log (n+1)$. If $f(n)$ denotes the number of representations of $n$ in the form $n=a_{k}+\mu_{l}$, where $a_{1}<a_{2}<\ldots<a_{k}<\ldots$ is a given sequence of integers such that $\lim _{k \rightarrow+\infty} \sqrt[k]{a_{k}}=e^{1 / a}$ exists, and $0<a<+\infty$, and $S_{r}$ is the sequence of those numbers $n$ for which $f(n)=r(r=0,1, \ldots)$, then $S_{r}$ has with probability 1 the density $(a b)^{r} e^{-a b} / r$ !.

Proof. The proof of Theorem 11 is analogous to that of Theorem 2. Putting $\varepsilon_{r}(n)=1$ or 0 according to whether $f(n)=r$ or $f(n) \neq r$, and taking into account that if $n^{\prime}$ is defined by $a_{n} \leqslant n<a_{n^{\prime}+1}$ then by supposition $n^{\prime} / \log n \rightarrow a$, we obtain

$$
\sum_{k=1}^{n^{\prime}} \frac{b}{\log \left(n-a_{k}+1\right)}=a b+\frac{b}{\log \left(n-a_{n^{\prime}}+1\right)}+o(1) .
$$

It follows that

$$
\lim _{N \rightarrow+\infty} \frac{1}{N} \sum_{n=1}^{N} M\left(\varepsilon_{r}(n)\right)=\frac{(a b)^{r} e^{-a b}}{r !} \quad(r=0,1, \ldots) .
$$

Taking into account that the number of quadruples $\left(n, m, a_{k}, a_{\imath}\right)$ with $n<m<N$ and $a_{k}<a_{l}<N$ such that $n-a_{k}=m-a_{l}$ is evidently of 
order $N \log ^{2} N$, we obtain

$$
D^{2}\left(\frac{1}{N} \sum_{n=1}^{N} \varepsilon_{r}(n)\right)=O\left(\frac{\log ^{2} N}{N}\right)
$$

and the proof can be completed as that of Theorem 3 .

Another possible stochastic analogon of Romanoff's theorem could be obtained by considering the sums $n=v_{k}+p$ where $v_{k}$ is a random sequence generated by the probabilities $p_{n}=c / n(c>0)$ (which implies according to (1.7) $\left.\lim _{k \rightarrow+\infty} \sqrt[k]{v_{k}}=e^{1 / c}\right)$ and $p$ is a prime. In that case, denoting by $f(n)$ the number of representations of $n$ in the form $n=v_{k}+p$, we have

$$
M(f(n))=\sum_{p<n} \frac{1}{n-p}
$$

where the summation is extended over all primes $p<n$; this sum is very sensitive to the irregularities of the distribution of primes and thus this problem requires careful separate consideration. If, however, we replace the sequence of primes by a sequence $\left\{\mu_{\}}\right\}$of similar growth but more regular behaviour, then these difficulties disappear.

We consider the case where both sequences are random in the following section.

\section{\$12. The sum of a dense and a rare sequence. In this section we} shall prove a general theorem, which is of the same type as Theorem 2 and which covers some cases in which condition $(2.5)$ is not satisfied. This theorem runs as follows.

THEOREM 12. Let $\left\{\boldsymbol{v}_{k}\right\}$ and $\left\{\mu_{l}\right\}$ be independent random sequences of integers, generated by the sequence of probabilities $\left\{p_{n}\right\}$ and $\left\{q_{n}\right\}$, where the sequence $q_{n}$ is decreasing. Let us put

$$
P(n)=\sum_{k=1}^{n} p_{k} \quad \text { and } \quad Q(n)=\sum_{k=1}^{n} q_{k}
$$

and suppose that $P(n) \rightarrow+\infty, Q(n) \rightarrow+\infty$ for $n \rightarrow+\infty$ and that for any $p>0$ we have

Further, let us put

$$
\lim _{n \rightarrow+\infty} \frac{Q(n p)}{Q(n)}=p
$$

$$
r_{n}=\sum_{k=1}^{n-1} p_{k} q_{n-k}
$$

and

$$
R(n)=\sum_{k=1}^{n} r_{k}
$$

Suppose that

$$
\lim _{n \rightarrow+\infty} \frac{R(n)}{n}=\lambda>0 .
$$

Let $f(n)$ denote the number of solutions of $n=v_{k}+\mu_{l}$; then the sequence $S_{r}$ of numbers $n$ for which $f(n)=r$ has with probability 1 the density $\lambda^{r} e^{-\lambda} / r$ ! $(r=0,1,2, \ldots)$.

Remark. Clearly it follows from (12.2) that condition (2.5) is not fulfilled. Thus Theorem 12 can be applied in cases where Theorem 2 is not applicable.

Proof of Theorem 12. For the proof we shall need some results of a Tauberian kind.

LEMiva 1. Let $d_{k}$ be a sequence of positive numbers, $a \geqslant 0$. Put

$$
D(y)=\sum_{k<y} d_{k}
$$

and

$$
d(x)=\sum_{k=1}^{\infty} d_{k} x^{k} .
$$

If the series (12.7) is convergent for $|x|<1$ and for every $p>0$ we have

then we have

and thus

$$
\lim _{x \rightarrow 1-0} \frac{d\left(x^{p}\right)}{d(x)}=\frac{1}{p^{\alpha}},
$$

Conversely if (12.10) holds then (12.7) is convergent for $|x|<1$ and (12.9) and thus (12.8) is valid. In other words, (12.8) and (12.10) are equivalent.

Remark. The first assertion of Lemma 1 is due to J. Karamata [17], the second to N. G. de Bruijn and P. Erdös [18]. 
LEMMA 2. Let $\left\{p_{k}\right\}$ and $\left\{q_{k}\right\}$ be two sequences of positive numbers, and put

$$
r_{k}=\sum_{k=1}^{n-1} p_{k} b_{n-k}
$$

Put

$$
P(n)=\sum_{k=1}^{n} p_{k}, \quad Q(n)=\sum_{k=1}^{n} q_{k}, \quad R(n)=\sum_{k=1}^{n} r_{k} .
$$

Suppose that for every $p>0$ we have

$$
\lim _{y \rightarrow+\infty} \frac{Q(p y)}{Q(y)}=p^{a} \quad \text { and } \quad \lim _{y \rightarrow+\infty} \frac{R(p y)}{R(y)}=p^{\gamma}
$$

where $0 \leqslant \alpha \leqslant \gamma$. Then we have

$$
\lim _{n \rightarrow+\infty} \frac{P(n) Q(n)}{R(n)}=\frac{\Gamma(1+\gamma)}{\Gamma(1+\alpha) \Gamma(1+\gamma-\alpha)} .
$$

Remark. Lemma 2 is due to N. G. de Bruijn and P. Erdös [18]. Now we are in a position to prove Theorem 12. Let us put

$$
\text { (12.15) } \quad p(x)=\sum_{k=1}^{\infty} p_{k} x^{k}, \quad q(x)=\sum_{k=1}^{\infty} q_{k} x^{k} \quad \text { and } \quad r(x)=\sum_{k=1}^{\infty} r_{k} x^{k} \text {. }
$$

Applying Lemma 1 (with $\alpha=1$ ) we obtain with respect to (12.2) and (12.5)

$$
\text { (12.16) } \quad \lim _{x \rightarrow 1-0} \frac{q\left(x^{p}\right)}{q(x)}=\frac{1}{p} \quad \text { and } \quad \lim _{x \rightarrow 1-0} \frac{r\left(x^{p}\right)}{r(x)}=\frac{1}{p} \text {. }
$$

Since by (12.3) we clearly have

$$
p(x) q(x)=r(x)
$$

it follows that

$$
\lim _{x \rightarrow 1-0} \frac{p\left(x^{p}\right)}{p(x)}=1 .
$$

Applying again Lemma 1 (with $\alpha=0$ ) we obtain

$$
\lim _{n \rightarrow+\infty} \frac{P(p n)}{P(n)}=1 \quad \text { for any } p>0 .
$$

Thus we can apply Lemma 2 with $\gamma=1$ and $a=0$ and obtain

$$
\lim _{n \rightarrow+\infty} \frac{P(n) Q(n)}{n}=\lambda \text {. }
$$

Denoting by $E(N)$ the number of values of $k$ for which $v_{k}<N$ we have, by (1.5), with probability 1

$$
\lim _{N \rightarrow+\infty} \frac{E(N)}{P(N)}=1
$$

Let $\left\{a_{k}\right\}$ be a fixed realization of the sequence $\left\{v_{k}\right\}$ for which (12.21) holds. Put

$$
t_{n}=\sum_{a_{k}<n} q_{n-a_{k}}
$$

and

$(12.23)$

$$
T(N)=\sum_{n=1}^{N} t_{n}
$$

Then we evidently have

$$
T(N)=\sum_{a_{k}<N} Q\left(N-a_{k}\right) .
$$

On the other hand, if $e_{n}=1$, if $n=a_{k}^{*}$ and $e_{n}=0$ if $a_{k}<n<a_{k+1}$, we have

(12.25)

$$
t_{n}=\sum_{k=1}^{n-1} q_{n-k} e_{k}
$$

Let us put

and

$(12.27)$

$$
e(x)=\sum_{k=1}^{\infty} e_{k} x^{k}
$$

By virtue of (12.21) and (12.19) we may apply Lemma 1 with $\alpha=0$ for the sequence $e_{k}$; we obtain

$$
\lim _{x \rightarrow 1-0} \frac{e\left(x^{p}\right)}{e(x)}=1
$$

Since by (12.27) we have
(12.29)
$t(x)=q(x) e(x)$,

it follows from (12.28) and the first relation of (12.16) that

$$
\lim _{x \rightarrow 1-0} \frac{t\left(x^{p}\right)}{t(x)}=\frac{1}{p} .
$$


Applying again (in the opposite direction) Lemma 1 with $\alpha=1$ for the sequence $t_{n}$, we find that for $p>0$

$$
\lim _{N \rightarrow+\infty} \frac{T(p N)}{T(N)}=p .
$$

Applying again Lemma 2, by (12.31) we have

$$
\lim _{n \rightarrow+\infty} \frac{E(n) Q(n)}{T(n)}=1,
$$

and thus with respect to (12.21) we obtain

$$
\lim _{n \rightarrow+\infty} \frac{P(n) Q(n)}{T(n)}=1
$$

and finally with respect to $(12.20)$

$$
\lim _{n \rightarrow+\infty} \frac{T(n)}{n}=\lambda .
$$

Now, since $q_{n}$ is decreasing, we clearly have

$$
Q(2 n)-Q(n)=\sum_{n<k \leqslant 2 n} q_{k} \leqslant n q_{n}
$$

and thus

$$
\liminf _{n \rightarrow+\infty} \frac{n q_{n}}{Q(n)} \geqslant \lim _{n \rightarrow+\infty} \frac{Q(2 n)-Q(n)}{Q(n)}=1 .
$$

On the other hand, we have by (12.25)

$$
\text { (12.37) } \quad t_{n} \geqslant q_{n} E(n)
$$

and thus by (12.20), (12.21) and (12.36)

$$
\text { (12.38) } \quad \liminf _{n \rightarrow+\infty} t_{n} \geqslant \lambda .
$$

Thus the sequence $t_{n}$, which according to $(12.34)$ is $(C, 1$.$) -summable$ to the limit $\lambda$, is such that its inferior limit is not less than $\lambda$. This clearly. implies

$$
\liminf _{n \rightarrow+\infty} t_{n}=\lambda \text {. }
$$

Now we need the following (well-known)

LEMMA 3. If a sequence $t_{n}$ is $(C, 1)$-summable to the limit $\lambda$ and liminf $t_{n}$ $=\lambda$, then for any bounded function $g(x)$ which is continuous for $x=\lambda$ the sequence $g\left(t_{n}\right)$ is $(C, 1)$-summable to the limit $g(\lambda)$.
Proof. The proof of Lemma 3 is very simple; as a matter of fact; our suppositions imply that for any $\varepsilon>0$ the sequence of those values of $n$ for which $t_{n} \geqslant \lambda+\varepsilon$ has the density 0 , whence - in view of the suppositions concerning $g(x)$ - the assertion of Lemma 3 immediately follows.

We can apply Lemma 3 to our sequence $t_{n}$ in view of (12.34) and (12.39) and for the continuous and bounded functions $g_{r}(x)=x^{r} e^{-x} / r$ ! $(r=0,1, \ldots)$, and find that

$$
\lim _{n \rightarrow+\infty} \frac{1}{n} \sum_{k=1}^{n} \frac{t_{k}^{r} e^{-t_{k}}}{r !}=\frac{\lambda^{r} e^{-\lambda}}{r !} \quad(r=0,1, \ldots) .
$$

Let us now define the random variables $\varepsilon_{r}(n)$ as follows: $\varepsilon_{r}(n)=1$ if $n$ has exactly $r$ representations of the form $n=a_{k}+\mu_{l}$; otherwise we put $\varepsilon_{r}(n)=0$. It follows that

(12.41) $M\left(\varepsilon_{r}(n)\right)=\sum_{i_{1}<i_{2}<\ldots<i_{r}} q_{n-a_{i_{1}}} q_{n-a_{i_{2}}} \ldots q_{n-a_{i_{r}}} \prod_{\substack{a_{k}<n \\ k \neq i_{1}, i_{2}, \ldots, i_{r}}}\left(1-q_{n-a_{k}}\right)$.

Thus we find by $(12.40)$ that

$$
\lim _{N \rightarrow+\infty} \frac{1}{N} \sum_{n=1}^{N} M\left(\varepsilon_{r}(n)\right)=\frac{\lambda^{r} e^{-\lambda}}{r !} \quad(r=0,1, \ldots) .
$$

Let us now consider the random variable

$$
\zeta_{r}(N)=\frac{1}{N} \sum_{n=1}^{N} \varepsilon_{r}(n) .
$$

It follows by (12.42) that

$$
\lim _{N \rightarrow+\infty} M\left(\zeta_{r}(N)\right)=\frac{\lambda^{r} e^{-\lambda}}{r !} \quad(r=0,1, \ldots) .
$$

Let us now consider the variance of $\zeta_{r}(N)$.

We evidently have

(12.45) $D^{2}\left(\zeta_{r}(N)\right)=\frac{1}{N^{2}}\left(\sum_{n=1}^{N} \sum_{m=1}^{N}\left[M\left(\varepsilon_{r}(n) \varepsilon_{r}(m)\right)-M\left(\varepsilon_{r}(n)\right) M\left(\varepsilon_{r}(m)\right)\right]\right)$.

Clearly $\varepsilon_{\mathbf{r}}(n), \varepsilon_{\mathbf{r}}(m)$ are independent if $n-m$ cannot be represented in the form $n-m=a_{k_{1}}-a_{k_{2}}$ because in that case $n-a_{k_{1}}$ and $m-a_{k_{2}}$ cannot be equal and thus a pair of representations $n=a_{k_{1}}+\mu_{l}$ and $m=a_{k_{2}}+\mu_{l}$ with the same $\mu_{l}$ is impossible. For a fixed pair $\left(k_{1}, k_{2}\right)$ the 
number of pairs $(n, m)$ for which $n \leqslant N, m \leqslant N$ and $n-m=a_{k_{1}}-a_{k_{2}}$ cannot exceed $N$, and the number of pairs $\left(k_{1}, \hat{k}_{2}\right)$ with $a_{k_{1}}<N, a_{k_{2}}<N$ does not exceed $C P^{2}(N)$ where $c>0$. In view of

it follows that

$$
\left|M\left(\varepsilon_{r}(n) \varepsilon_{r}(m)\right)-M\left(\varepsilon_{r}(n)\right) M\left(\varepsilon_{r}(m)\right)\right| \leqslant 1
$$

Clearly for any $\varepsilon>0$ we have by (12.19)

$$
\frac{P(2 n)}{P(n)} \leqslant 1+\varepsilon \quad \text { for } \quad n \geqslant n_{0}(\varepsilon) .
$$

This implies that for any $\delta>0$

$$
P(N)=O\left(N^{\delta}\right)
$$

Thus we obtain from (12.46) and (12.48)

$$
D^{2}\left(\zeta_{r}(N)\right)=O\left(\frac{1}{N^{1-\delta}}\right) \quad \text { for any } \delta>0 .
$$

Now, exactly as in proving Theorem 2, we conclude that with probability 1 we have

$$
\lim _{N \rightarrow+\infty} \zeta_{r}(N)=\frac{\lambda^{r} e^{-\lambda}}{r !} .
$$

Thus the sequence $S_{r}$ of those integers which have exactly $r$ representations of the form $n=a_{k}+\mu_{l}$ where $\left\{a_{k}\right\}$ is a fixed realization of the random sequence $\left\{v_{k}\right\}$, for which (12.21) holds, has with probability 1 the density $\lambda^{r} e^{-\lambda} / r$ !

Since almost every realization $\left\{a_{k}\right\}$ of the random sequence $\left\{v_{k}\right\}$ satisfies (12.21), it follows that the sequence $S_{r}$ of those integers $n$ which have exactly $r$ representations of the form $n=\nu_{k}+\mu_{l}$ has with probability 1 the density $\lambda^{r} e^{-\lambda} / r$ !.

To show that this last - intuitively clear - step is really admissible, let us consider the following realization of the probabilistic set-up. Let the probability space be the unit square of the $(x, y)$ plane, and let the probability measure $P$ be the ordinary Lebesgue plane measure. A random variable is then nothing else than a measurable function $\zeta(x, y)$ of the two variables $x$ and $y(0 \leqslant x \leqslant 1,0 \leqslant y \leqslant 1)$. Let us construct the random variables $\xi_{n}$ so that they depend only on $x$, i. e. $\xi_{n}(x, y)=$ $\xi_{n}(x)$ and the random variables $\eta_{n}$ so that they depend only on $y$, i.e. $\eta_{n}(x, y)=\eta_{\dot{n}}(y)$; suppose that the $\xi_{n}(x)$ are independent (with respect to the Lebesgue measure in the interval $0 \leqslant x \leqslant 1$ ) and, further, that the $\eta_{n}(y)$ are also independent (with respect to the Lebesgue measure in the interval $0 \leqslant y \leqslant 1)$ ); suppose that they have the preseribed distributions, i. e. $\xi_{n}(x)=1$ on a set of measure $p_{n}$ and 0 elsewhere and, further $\eta_{n}(y)=1$ on a set of measure $q_{n}$ and 0 elsewhere. Then for each point $(x, y)$ of the square $0 \leqslant x \leqslant 1,0 \leqslant y \leqslant 1$ the sequences $v_{k}$ and $\mu_{l}$ are well-defined (numerical) sequences of positive integers and thus the sequence $S_{r}=S_{r}(x, y)$ is also uniquely defined for all points $(x, y)$. Now let $E_{r}$ denote the set of those points $(x, y)$ for which the sequence $S_{r}$ has the density $\lambda^{r} e^{-\lambda} / r$ ! and let $\bar{E}_{r}$ be the complementary set. According to Theorem 11 the intersection of $\bar{E}_{r}$ with the line $x=x_{0}\left(0 \leqslant x_{0} \leqslant 1\right)$ has for almost all $x_{0}$ the (linear) measure 0 ; thus by Fubini's theorem $\bar{E}_{r}$ has itself the plane measure 0 , which was to be proved.

Thus Theorem 12 is proved.

Remark. If $p_{n}=a / n(a>0)$ and $a_{n}=b$ tog $(n+1) \quad(b>0)$, then all conditions of Theorem 12 are satisfied (with $\lambda=a b$ ) and thus the conclusion of Theorem 12 is valid. Note that in that case, according to (1.7), we have with probability $1 \lim _{k \rightarrow+\infty} \sqrt[k]{v_{k}}=e^{1 / a}$, and denoting by $\Pi(N)$ the number of terms of the sequence $\mu_{l}$ not exceeding $N$ we have by (1.5) with probability $1 \Pi(N) \sim b N / \log N$. Thus this special case of Theorem 12 furnishes another stochastic analogon of Romanoff's theorem.

It would be possible to prove a more general theorem, which includes both Theorem 2 and Theorem 12, but to obtain the most general theorem one has to solve some difficulties of Tauberian type.

\section{References}

[1] E. Landau, Handbuch der Lehre der Verteilung der Primzahlen, Bd. II. p. 643 .

[2] Unpublished dissertation (University of Cambridge), (oral communication of the author).

[3] P. Erdös, Problems and results in additive number theory, Colloque sur la théorie des nombres, Bruxelles 1955, p. $127-137$.

[4] S. Sidon, Über die Fourier Konstanten der Funktionen der Klasse $L_{p}$ für $p>1$, Acta Sci. Math. Szeged 7 (1935), p. 175-176.

[5] - Ein Satz über trigonometrische Polynome und seine Anwendungen in der Theorie der Fourier-Reihen, Math. Annalon, 106 (1932), p. 539.

[6] N. P. Romanoff, Über einige Sätze der additiven Zahlentheorie, Math. Annalen 109 (1934), p. 668-678.

[7] P. Erdös and A. Rényi, Some further statistical properties of the digits in Cantor's series, Acta Math. Ac. Sei. Hung. 10 (1959), p. $21-29$.

[8] B. V. Gnedenko and A. N. Kolmogorov, Limit distributions for sums of independent random variables, Addison-Wesley, 1954. 
[9] A. Kolmogoroff, Grundbegriffe der Wahrscheinlichleitsrechnung, Springer, Berlin, 1933.

[10] A. Rényi, Valószinüségszámitás (Textbook of Probability theory), Budapest, Tankönyvkiadó, 1954

[11] P. Erdös and P. Turán, On a problem of Sidon in additive number theory and on some related problems, Journal London Math. Soc. 16 (1941), p. $212-215$.

[12] S. Kakutani, On equivalence of infinite product measures, Annals of Math. 49 (1948), p. $214-224$

[13] A. Stöhr, Gelöste und ungelöste Fragen über Basen der natürlichen Zahlen, Journal für reine und angew. Math. 194 (1955), p. 40-65, $111-140$.

[14] P. Erdös, On some problems of Bellmann and a theorem of Romanoff, Journal of the Chinese Math. Soc. (1951), p. 409-421.

[15] G. H. Hardy and E. M. Wright, An introduction to the theory of numbers, 3rd. edition, Oxford, 1954 .

[16] P. Erdös and A. Rényi, On Cantor's series with convergent $\Sigma 1 / q_{n}$, Anna. les Univ. R. Eötvös. Budapest, Sect. Math. 2 (1959) (in print).

[17] J. Karamata, Neuer Beweis und Verallgemeinerung der Tauberschen Sätze, welche die Laplacesche und Stieltjessche Transformation betreffen, J. reine u. angew. Math. 164 (193I), p. 27.39.

[18] N. G. de Bruijn and P. Erdös, On a recursion formula and on some Tauberian theorems, Journal of Research of the Nat. Bureau of Stand. 50 (1953), p. 161 . 164 .

Reģu par la Rédaction le 28. 10. 1959

\section{A sum involving the function of Möbius}

by

D. H. Lehmer and S. Selberg (Berkeley, Cal., and Trondheim, Norway)

Let $\mu(n)$ be the Möbius function. The sum

$$
g(x)=\sum_{n \leqslant x} \mu(n) / n
$$

may itself be summed to give

$$
G(x)=\sum_{n \leqslant x} g(n)
$$

In this note we show that $G(x)-2$ changes sign infinitely often. Some numerical calculations of the first 56 sign changes are described. These show that these "zeros" of $G(x)-2$ are remarkably close to being in geometric progression with two exceptions. An heuristic explanation of this phenomenon is given.

It is equally easy to show that for any real $K$, the function $G(x)-K$ changes sign infinitely often. For this purpose we may treat $G(x)$ as a continuous, piecewise linear, function defined for $n \leqslant x<n+1$ by

$$
G(x)=G(n)+(x-n) g(n+1) .
$$

Let $s=\sigma+i t$ and suppose there is a $u_{0}$ such that $G(u)-K$ is of fixed sign for all $u \geqslant u_{0}$. For $\sigma>1$ we may write

$$
\begin{aligned}
\int_{u_{0}}^{\infty} \frac{G(u)-\mathbf{K}}{u^{s+1}} d u & =-\int_{1}^{u_{0}} \frac{G(u)-K}{u^{s+1}} d u+\int_{1}^{\infty} \frac{G(u)-K}{u^{s+1}} d u \\
& =\int_{1}^{\infty} \frac{G(u)}{u^{s+1}} d u+\frac{1}{s} f(s)
\end{aligned}
$$

where $f(s)$ is regular for $\sigma>0$. Using (1) we have, with $\sigma>1$,

(2) $\int_{u_{0}}^{\infty} \frac{G(u)-K}{u^{s+1}} d u=\frac{1}{s} f(s)+\frac{1}{s} \sum_{n=1}^{\infty} \frac{g(n)}{n^{s}}+\sum_{n=1}^{\infty} g(n+1) \int_{0}^{1} \frac{\Theta d \Theta}{(n+\Theta)^{s+1}}$. 